\title{
Threshold hyperon production in proton-proton collisions at COSY-11
}

\author{
T. Rożek ${ }^{\mathrm{a}, \mathrm{e}, *}$, D. Grzonka ${ }^{\mathrm{a}}$, H.-H. Adam ${ }^{\mathrm{b}}$, A. Budzanowski ${ }^{\mathrm{c}}$, R. Czyżykiewicz $^{\mathrm{d}}$, M. Janusz ${ }^{\mathrm{d}}$, \\ L. Jarczyk ${ }^{d}$, B. Kamys ${ }^{d}$, A. Khoukaz ${ }^{\text {b }}$, K. Kilian ${ }^{a}$, P. Klaja ${ }^{d}$, P. Kowina ${ }^{\mathrm{a}, \mathrm{e}}$, P. Moskal ${ }^{\mathrm{a}, \mathrm{d}}$, \\ W. Oelert ${ }^{a}$, C. Piskor-Ignatowicz ${ }^{\mathrm{d}}$, J. Przerwa ${ }^{\mathrm{d}}$, J. Ritman ${ }^{\mathrm{a}}$, T. Sefzick ${ }^{\mathrm{a}}$, M. Siemaszko ${ }^{\mathrm{e}}$, \\ J. Smyrski ${ }^{\text {d }}$, A. Täschner ${ }^{\text {b }}$, P. Winter ${ }^{\text {a }}$, M. Wolke ${ }^{\text {a }}$, P. Wüstner ${ }^{\mathrm{f}}$, Z. Zhang ${ }^{\text {a }}$, W. Zipper ${ }^{\mathrm{e}}$ \\ a Institut für Kernphysik, Forschungszentrum Jülich, D-52425 Jülich, Germany \\ b Institut für Kernphysik, Westfälische Wilhelms-Universität, D-48149 Münster, Germany \\ c Institute of Nuclear Physics, PL-31-342 Cracow, Poland \\ $\mathrm{d}$ Institute of Physics, Jagellonian University, PL-30-059 Cracow, Poland \\ ${ }^{\mathrm{e}}$ Institute of Physics, University of Silesia, PL-40-007 Katowice, Poland \\ ${ }^{\mathrm{f}}$ Zentrallabor für Elektronik, Forschungszentrum Jülich, D-52425 Jülich, Germany \\ Received 26 September 2005; received in revised form 28 June 2006; accepted 15 July 2006 \\ Available online 10 August 2006 \\ Editor: V. Metag
}

\begin{abstract}
The $\Sigma^{+}$hyperon production was measured at the COSY-11 spectrometer via the $p p \rightarrow n K^{+} \Sigma^{+}$reaction at excess energies of $Q=13 \mathrm{MeV}$ and $Q=60 \mathrm{MeV}$. These measurements continue systematic hyperon production studies via the $p p \rightarrow p K^{+} \Lambda / \Sigma^{0}$ reactions where a strong decrease of the cross section ratio close-to-threshold was observed. In order to verify models developed for the description of the $\Lambda$ and $\Sigma^{0}$ production we have performed the measurement on the $\Sigma^{+}$hyperon and found unexpectedly that the total cross section is by more than one order of magnitude larger than predicted by all anticipated models. After the reconstruction of the kaon and neutron four momenta, the $\Sigma^{+}$is identified via the missing mass technique. Details of the method and the measurement will be given and discussed in view of theoretical models.
\end{abstract}

(C) 2006 Elsevier B.V. All rights reserved.

PACS: $13.60 . \mathrm{Hb} ; 13.75 .-\mathrm{n} ; 25.40 . \mathrm{Ve} ; 28.20 .-\mathrm{n}$

Keywords: Strangeness; Kaon; Near threshold hyperon production; Sigma production; COSY-11

\section{Introduction}

The study of the hyperon production in hadron induced multi particle exit channels like $p p \rightarrow N K Y$ includes several aspects. The nucleon-hyperon interaction can be extracted by analyzing the $N Y$ subsystem in the appropriate kinematical region. Closely related to that is the issue of the reaction mechanisms of the hyperon production which have to be clarified for an unambiguous interpretation of the data. If the hyperon production is due to the excitation and a subsequent decay of

\footnotetext{
* Corresponding author.

E-mail address: trozek@us.edu.pl (T. Rożek).
}

intermediate nucleon resonances it allows to extract information about the structure of the relevant resonances.

The $p p \rightarrow p K^{+} \Lambda$ excitation function close-to-threshold shows a clear deviation from the pure phase space distribution and a proton-hyperon final state interaction (FSI) has to be included to describe the data [1-4]. In the $p p \rightarrow p K^{+} \Sigma^{0}$ channel the $p Y$ FSI seems to be negligible and the pure phase space calculations follow reasonably well the data points. The cross section ratio $\sigma\left(p p \rightarrow p K^{+} \Lambda\right) / \sigma\left(p p \rightarrow p K^{+} \Sigma^{0}\right)$ below excess energies of $Q \sim 20 \mathrm{MeV}$ is in the order of $28[2,3]$ in contrast to the value of about 2.5 determined for excess energies higher than $Q=300 \mathrm{MeV}$ [5] (see Fig. 1). This value is in good agreement with the $\Lambda / \Sigma^{0}$ isospin relation. The question arises if this drastic cross section increase close-to-threshold is 


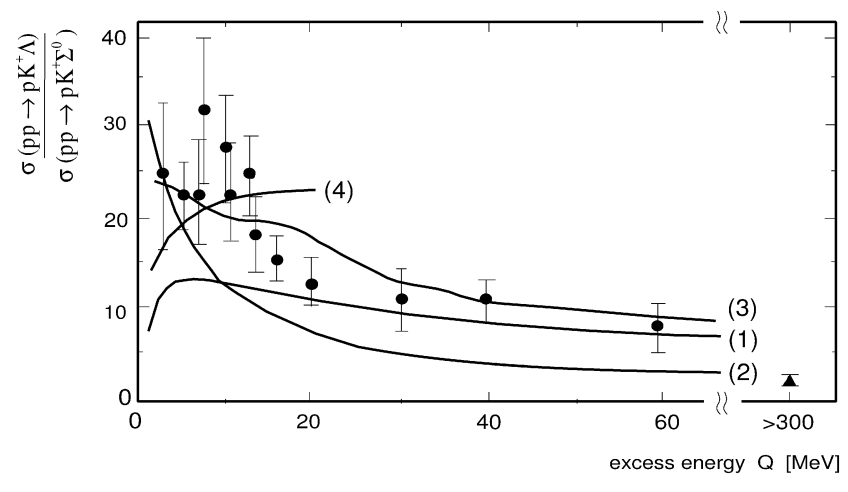

Fig. 1. The cross section ratio for $\Sigma^{0}$ and $\Lambda$ production in the threshold region. The data [1-3] are compared to different model predictions. The high energy data point is taken from Ref. [5]. The curves are theoretical predictions described in the text.

a mere effect of the $N \Lambda$ FSI or whether it is partly due to the reaction mechanisms in the $N Y$ channels. To explain the increase of the $\Lambda / \Sigma^{0}$ cross section ratio in the close-to-threshold region, different scenarios were proposed.

Calculations of the strangeness production by solely $\pi$ and $K$ exchange added incoherently have been performed in Ref. [6]. The $\pi N \rightarrow Y K$ and $K N \rightarrow Y K$ scattering amplitudes for pion and kaon exchange, respectively, were taken from the existing data in the higher energy region [5]. Since this incoherent $\pi / K$ exchange model describes the $p p \rightarrow p K^{+} \Lambda$ cross section over the whole energy range, but overestimates the close-to-threshold region in case of the $p p \rightarrow p K^{+} \Sigma^{0}$ channel, the predicted $\Lambda / \Sigma^{0}$ ratio is too low for $Q \leqslant 20 \mathrm{MeV}$ (see curve (1) in Fig. 1).

A better description of the strong rise of the ratio towards lower $Q$-values is achieved by the resonance model (curve (2) in Fig. 1) [6-9]. In this model the nonresonant direct contributions like $\pi$ or $K$ exchange were not included, but the $\pi, \eta$ and $\rho$ meson exchange with the excitation of the intermediate baryonic resonances $N(1650), N(1710), N(1720)$ and $\Delta(1920)$ are taken into account. In this resonance model the close-to-threshold region of the $\Lambda / \Sigma^{0}$ cross section ratio seems to be better reproduced than the higher energy values i.e. ( $Q \geqslant 10 \mathrm{MeV}$ ). It should be stressed that in these calculations the parameters were fixed on the basis of higher energy data, before the close-to-threshold $\Lambda$ and $\Sigma^{0}$ data were available.

Other calculations by Shyam [10] (based on the effective Lagrangian model) include for the strangeness production also meson exchange $(\pi, \rho, \sigma$ and $\omega)$ together with the excitation of resonances. The coupling constant was determined by fitting data of the $\pi^{+} p \rightarrow \Sigma^{+} K^{+}, \pi^{-} p \rightarrow \Sigma^{0} K^{0}$ and $\pi^{-} p \rightarrow$ $\Sigma^{-} K^{+}$reaction channels. The coherent sum of resonant states and meson exchange processes describe the experimental data for the $p p \rightarrow p K^{+} \Lambda$ and $p p \rightarrow p K^{+} \Sigma^{0}$ channels very well. The effective Lagrangian model is depicted by the curve (3) in Fig. 1.

The Jülich theory group has performed calculations including $\pi$ and $K$ exchange $[11,12]$. In their approach the interaction between the hyperons $(\Lambda, \Sigma)$ and the nucleon is described by a microscopic $(\Lambda N-\Sigma N)$ coupled channel model [13] with a coherent superposition of the production amplitudes. The $\Lambda$ production is dominated by the $K$ exchange and therefore the contribution due to an interference between $\pi$ and $K$ exchange is negligible in this hyperon channel. On the other hand the $\pi$ and $K$ exchanges give a comparable contribution to the cross section in the case of $\Sigma^{0}$ production. An interference between $\pi$ and $K$ exchange amplitudes act very differently on the two channels. Within the Jülich meson exchange model the large cross section ratio can be described by a destructive interference of the $\pi$ and $K$ exchange amplitudes only. For excess energies above $20 \mathrm{MeV}$ the model is not valid any more but qualitatively the cross section ratio given by the model stays at a nearly constant level.

Although the various descriptions of the cross section ratio differ even in the dominant reaction mechanism, all reproduce more or less the trend of an increase of the $\Lambda / \Sigma^{0}$ cross section ratio in the threshold region (see Fig. 1). The present data are not sufficient to definitely exclude possible explanations and therefore an unambiguous identification of the dominant reaction mechanism is impossible. To clarify this point further data are needed. Especially the other isospin channels should allow to extract information about the production mechanisms. Recently, besides the $\Lambda$ and $\Sigma^{0}$ production the reaction channel $p p \rightarrow n K^{+} \Sigma^{+}$became accessible at the COSY-11 detection system after the installation of a neutron detector. The measurement of the $\Sigma^{+}$hyperon production via this reaction was performed at two beam momenta, $P_{\text {beam }}=2.6 \mathrm{GeV} / c$ and $P_{\text {beam }}=2.74 \mathrm{GeV} / c$, corresponding to excess energies of $13 \mathrm{MeV}$ and $60 \mathrm{MeV}$, respectively.

\section{Experiment}

COSY-11 is an internal magnetic spectrometer at the COoler SYnchroton and storage ring COSY [14] in Jülich. The interaction between a proton in the beam and a proton from the $\mathrm{H}_{2}$ cluster target [15] may lead to the production of the $\Sigma^{+}$ hyperon in the $p p \rightarrow n K^{+} \Sigma^{+}$reaction. The charged reaction products are separated from the circulating beam in the magnetic field of one of the regular COSY dipoles [16]. The $\Sigma^{+}$ hyperon is identified via the missing mass technique by detecting the $K^{+}$and the neutron. The momentum vector of the $K^{+}$ meson can be established by tracking back the $K^{+}$trajectory reconstructed in the drift chambers (DC1 and DC2 in Fig. 2) through the known magnetic field back to the target point. Together with the velocity measurement in the two scintillators S8 and $\mathrm{S} 1$, the kaon is identified via its invariant mass.

Assuming a hit in the neutron detector being due to a neutron, the four momentum vector of the neutron is given by the measured velocity, the direction of the neutron (given by the first hitted module) and the known mass. The background from charged particles hitting the neutron detector is discriminated by veto scintillators.

In Fig. 3 the experimental distributions of the squared missing mass $\left(m_{x}^{2}\right)$ of the $p p \rightarrow n K^{+} X$ system for the two beam momenta are shown. For the higher momentum, an enhancement around the squared $\Sigma^{+}$mass is clearly seen on a large 


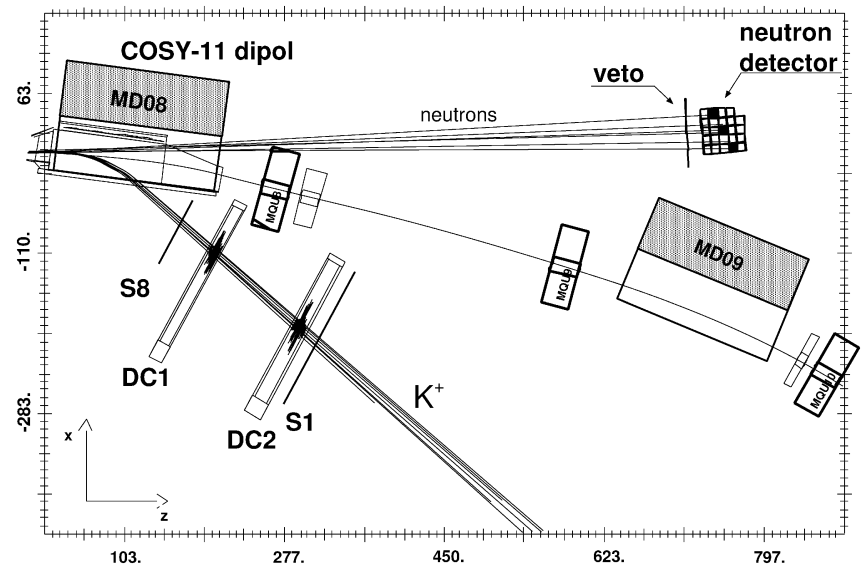

Fig. 2. COSY-11 detection set-up [16] with the superimposed tracks of kaons and neutrons from the $p p \rightarrow n K^{+} \Sigma^{+}$. The scale of the axes is given in [cm]. (a)

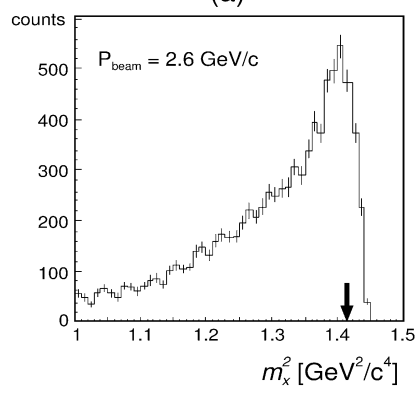

(b)

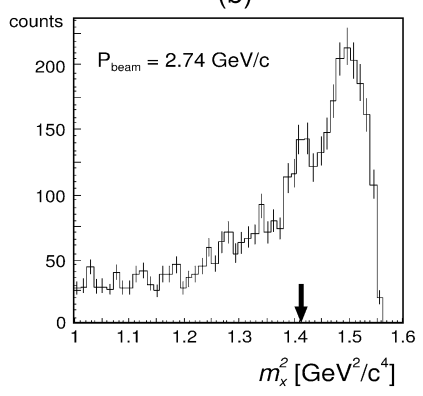

Fig. 3. The experimental squared missing mass of the $p p \rightarrow n K^{+} X$ system for two investigated beam momenta. The arrows point to the nominal squared mass of the $\Sigma^{+}$hyperon. The statistical errors of the counting rates are shown.

background (Fig. 3b), but for the lower beam momentum (Fig. 3a) a $\Sigma^{+}$peak is not directly visible.

In order to determine the number of $\Sigma^{+}$events in the higher energy data set, a fit has been done with a polynomial function superposed by the expected missing mass distribution of the $n K^{+}$system for the $p p \rightarrow n K^{+} \Sigma^{+}$reaction obtained from the simulation studies. In Fig. 4a the experimental missing mass spectrum of the $p p \rightarrow n K^{+} X$ system is compared with the fitted polynomial function. The expected distribution from MC studies with $X=\Sigma^{+}$is depicted in the figure as well. Fig. 4b shows the result of the subtraction of the fitted polynomial from the experimental missing mass distribution together with the MC distribution.

In order to understand the background distribution, 22 reaction channels (mostly multi-pion reactions but also $p p \rightarrow$ $\left.p K^{+} \Lambda\left(\Sigma^{0}, \Lambda \gamma\right)\right)$ were simulated and their contributions to the missing mass distribution were determined. These studies showed that the reactions $p p \rightarrow p K^{+} \Lambda$ and $p p \rightarrow p K^{+} \Lambda \gamma$ $\left(\gamma\right.$ 's) are the dominant background channels in the $\Sigma^{+}$region. The Monte Carlo code includes the realistic geometry and physics processes like energy loss and straggling which occasionally cause the misidentification of the particle type.

All background channels result in a rather smooth distribution of the missing mass spectrum as can be inferred from calculations from MC studies and by comparing the two experimental distributions (see Fig. 3). (a)

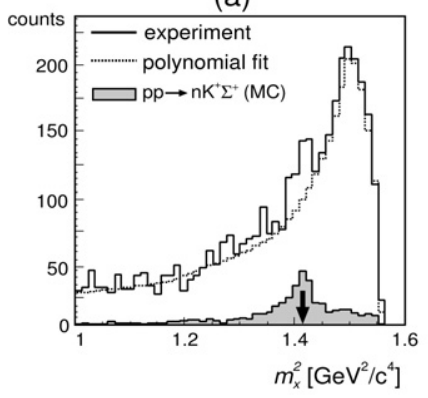

Fig. 4. Background determination for the $p p \rightarrow n K^{+} \Sigma^{+}$reaction at $P_{\text {beam }}=2.74 \mathrm{GeV} / c$. (a) The experimental squared missing mass spectrum of $n K^{+}$system with a polynomial background fit and the simulated $p p \rightarrow n K^{+} \Sigma^{+}$spectrum. (b) Result of the subtraction of the fitted background from the experimental distribution compared with the simulated spectrum. The arrows show the nominal squared mass of the $\Sigma^{+}$hyperon. Only statistical errors are shown.

For the lower energy data set a $\Sigma^{+}$peak is not obviously visible via the missing mass distribution. Therefore, a simple polynomial background fitting cannot be used. To determine the number of $\Sigma^{+}$events it was assumed that the background shape for this data set is the same as that at the higher energy. This assumption is justified since there is no new open channel for the higher energy.

At the COSY-11 experiment the shape of the missing mass distribution is mainly determined by the acceptance of the detection system and is dependent on the excess energy of an individual event. From the analysis of $\eta$ and $\eta^{\prime}$ production studies at COSY-11 it was verified that the background shape resulting here mainly from multi pion production is in very good agreement with the expectations from Monte Carlo studies taking into account the detector characteristics and is comparable at different beam momenta. In addition Monte Carlo data of the reaction channels which contribute dominant to the background in the $\Sigma^{+}$production were compared in view of the background shape by adjusting the kinematical limits. Within error bars their shapes were identical. Therefore it is justified to assume that the background shape is the same for both beam momenta. For a detailed discussion on the background shape at COSY-11 we refer to [17]. The background shape from the experimental missing mass distribution for the higher energy data set was determined and shifted such that the kinematical limits for both spectra were the same. Afterwards, it was fitted together with the missing mass distribution of the $p p \rightarrow n K^{+} \Sigma^{+}$ from $\mathrm{MC}$ studies to the experimental missing mass spectrum for the lower energy (see Fig. 5).

\section{Results}

\subsection{Total cross section}

For the lower energy data set, even after applying all cuts, there was no clear enhancement around the kaon mass in the invariant mass distribution, and therefore the assumption was made, that the kaon peak in the experimental distribution has the same position and width as in the simulated distribution. 
(a)

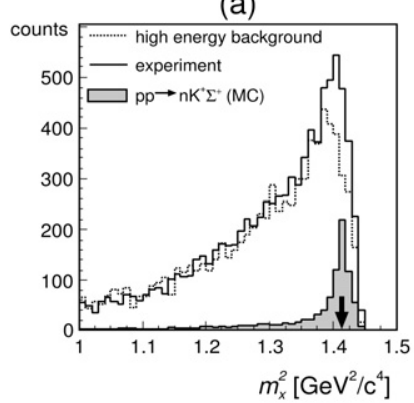

(b)

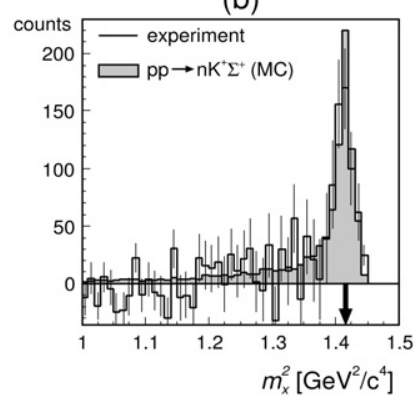

Fig. 5. Background investigation for the $p p \rightarrow n K^{+} \Sigma^{+}$reaction at $P_{\text {beam }}=2.6 \mathrm{GeV} / c$. (a) The experimental squared missing mass spectrum of the $p p \rightarrow n K^{+} X$ system compared to the assumed background taken from the data at $Q=60 \mathrm{MeV}$ and the simulated $p p \rightarrow n K^{+} \Sigma^{+}$distribution. (b) Result of the subtraction of the fitted background from the experimental distribution compared to the simulated spectrum. The arrows show the nominal squared mass of the $\Sigma^{+}$hyperon. Only statistical errors are shown.

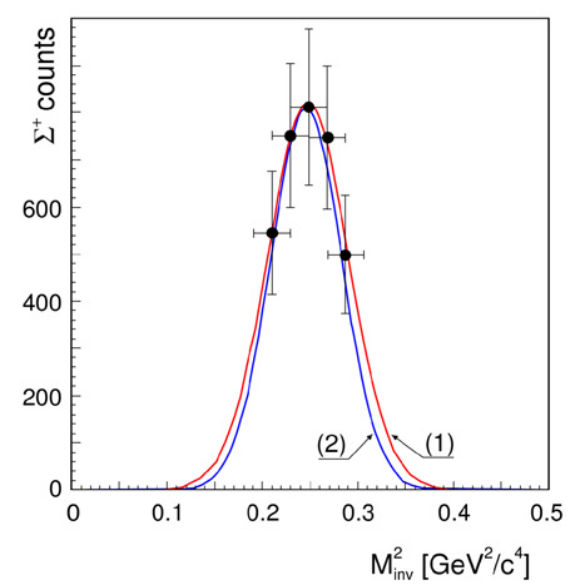

Fig. 6. The number of $\Sigma^{+}$events obtained for different regions of the invariant mass cut. Curve (1)—expected from the MC studies distribution of the $K^{+}$ events. Curve (2)-Gaussian fit to the points on the figure given by the number of $\Sigma^{+}$events. See text for details.

As a cross check, event samples with cuts on different regions but still within the kaon range in the experimental invariant mass distribution were taken and the missing mass was determined. The assumed background shape was subtracted from the experimental distribution and the enhancement around the mass of the $\Sigma^{+}$was interpreted as a signal from the $\Sigma^{+}$ hyperon (see Fig. 5). This procedure was repeated for five different invariant mass regions and the number of events under the remaining peak was determined. The results are shown in Fig. 6. The vertical error bars correspond to the statistical error of the number of events. The horizontal error bars show the widths of the appropriate invariant mass cuts which have been chosen as half of the standard deviation of the kaon peak for the data sample of $p p \rightarrow n K^{+} \Sigma^{+}$channel simulated with lower beam momentum. In Fig. 6 curve (1) shows the expected invariant mass distribution of kaons from MC calculations. Curve (2) is a Gauss fit to the experimentally determined number of $\Sigma^{+}$ events in the different invariant mass regions. This can be interpreted as an experimental distribution of kaons. The assumed

Table 1

The number of $\Sigma^{+}$events for the higher energy case. Only statistical errors are given

\begin{tabular}{lll}
\hline $\begin{array}{l}\text { Invariant } \\
\text { mass cut }\end{array}$ & $\begin{array}{l}\text { Number } \\
\text { of events } \\
\text { identified as } \Sigma^{+}\end{array}$ & $\begin{array}{l}\text { Number of events identified } \\
\text { as } \Sigma^{+} \text {and scaled to the full } \\
\text { Gaussian distribution }\end{array}$ \\
\hline $\begin{array}{l}\mu_{\exp } \\
\pm 0.25 \sigma_{\exp }\end{array}$ & $187 \pm 51$ & $944 \pm 257$ \\
$\pm 0.5 \sigma_{\exp }$ & $367 \pm 83$ & $960 \pm 217$ \\
$\pm 1.0 \sigma_{\exp }$ & $661 \pm 134$ & $969 \pm 197$ \\
\hline
\end{tabular}

Table 2

Values of the total cross section for the $p p \rightarrow n K^{+} \Sigma^{+}$reaction obtained in the present work. Both, statistical and systematical errors are presented

\begin{tabular}{lll}
\hline Beam momentum & Excess energy & Total cross section \\
$P_{\text {beam }}[\mathrm{GeV} / c]$ & $Q[\mathrm{MeV}]$ & $\sigma[\mu \mathrm{b}]$ \\
\hline 2.60 & 13 & $4.56 \pm 0.94 \pm 2.7$ \\
2.74 & 60 & $44.8 \pm 10.7 \pm 15.2$ \\
\hline
\end{tabular}

experimental kaon invariant mass distribution is in line with the distribution expected from MC studies.

For the higher beam momentum a kaon peak on the invariant mass distribution is clearly visible and can be fitted by a Gaussian function. In a systematical study of the result at the higher beam momentum three different cuts on the experimental invariant mass distribution were applied. Events from these regions, namely: $\mu_{\exp } \pm 0.25 \sigma_{\exp }, \mu_{\exp } \pm 0.5 \sigma_{\text {exp }}$ and $\mu_{\exp } \pm 1.0 \sigma_{\text {exp }}$ were taken and the corresponding missing mass distributions were generated. Next the number of $\Sigma^{+}$events for each of these distributions was determined. The results are listed in the middle column of Table 1 and in the last column the number of the $\Sigma^{+}$events corresponding to the full Gaussian distribution.

In order to calculate the cross section for the $p p \rightarrow n K^{+} \Sigma^{+}$ reaction the number of $\Sigma^{+}$hyperon events and the detection efficiency of the COSY-11 apparatus for the two excess energies were determined. The luminosity was determined by a simultaneous measurement of proton-proton elastic scattering.

In Table 2 the total cross sections for both beam momenta are given. The systematical errors are due to: (i) error of the detection efficiency determination which is $8.5 \%$ for the lower and $3.5 \%$ for the higher energy data set (including the inaccuracy of the effective detector position and of the beam momentum determination), (ii) uncertainty in the form of the background, and (iii) error of the luminosity calculation which is $3 \%$ for both data sets and includes the uncertainty due to the normalization procedure and the error of the solid angle determination. For the data at $2.74 \mathrm{GeV} / c$ the uncertainty in the background form was estimated by comparing the polynomial fit with a background form resulting from an adjusted sum of known background reaction channels generated in Monte Carlo studies. The difference is about $18 \%$. For the data at $2.6 \mathrm{GeV} / c$ the region for the adjustment of the background used for the subtraction was varied resulting in an error of about $20 \%$. The values in Table 2 include also a change in the detection efficiency resulting from the inclusion of higher partial waves. Close to the reaction threshold higher than $S$ partial wave contributions are 
not expected, however, if the excess energy $Q$ for the studied channel go beyond a few $\mathrm{MeV}$ range, higher partial waves can contribute to the production mechanism. Since in the case of the $\Sigma^{+}$hyperon production this contribution is unknown, its effect is assumed on the basis of the $p p \rightarrow p K^{+} \Lambda$ channel studied at the TOF experiment at COSY [18-20]. Higher partial waves in a strength given in $[18,19]$ result in a decrease of the detection efficiency by $30 \%$ for the lower and by $7.7 \%$ for the higher energy data set. The sum of the total systematical errors equals to $60 \%$ for the lower and $34 \%$ for the higher energy data set.

\subsection{Comparison with model predictions}

Among the models described in the introduction only two give predictions for the $p p \rightarrow n K^{+} \Sigma^{+}$reaction, namely the Jülich meson exchange model [11,12] and the resonance model [6,9]. Calculations of the $\Sigma^{+}$production within the Jülich meson exchange model predict a total cross section of $\sigma=0.23 \mu \mathrm{b}$ at $Q=13 \mathrm{MeV}$ for the destructive interference (which was necessary to describe the high $\Lambda / \Sigma^{0}$ cross section ratio at threshold). This is about a factor of 20 below the experimental value of $4.56 \mu \mathrm{b}$ given in Table 2 . A constructive interference would result in a cross section even a factor of 53 too low.

For the resonance model the predictions for the $p p \rightarrow$ $n K^{+} \Sigma^{+}$channel for the close-to-threshold region deviate even more from the data. In Fig. 7 the model predictions and the available data for the $p p \rightarrow n K^{+} \Sigma^{+}$(a), $p p \rightarrow p K^{+} \Sigma^{0}$ (b) and $p p \rightarrow p K^{+} \Lambda$ (c) channels are shown. The data points presented by triangles, dots and squares in the close-to-threshold region were measured by the COSY-11 Collaboration [1-3]. The data point in the $p p \rightarrow p K^{+} \Lambda$ channel indicated by the arrow was determined in parallel by selecting the $K^{+} p$ exit channel which was included in the triggered events from the $\Sigma^{+}$production data at $2.74 \mathrm{GeV} / c$. The high energy data for the given reactions were taken from [5] and [21]. The model calculations for each channel are given by the solid line [9,22].

The data point for the $p p \rightarrow n K^{+} \Sigma^{+}$channel at $Q=$ $13 \mathrm{MeV}$ is underestimated in the total cross section calculated using the resonance model $[6,9]$ by about a factor of 500 and for

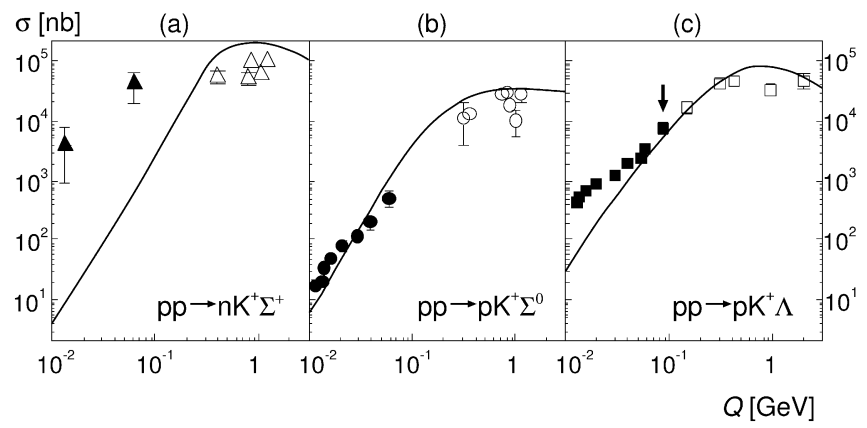

Fig. 7. Comparison of the experimental total cross section with the resonance model [6,9] predictions for various $p p \rightarrow N K^{+} Y$ reactions. Full triangles in (a) are data obtained in this work. Data in the close-to-threshold region (presented as full symbols in (b) and (c)) are taken from Refs. [1-3] and data from the high excess energy region (open symbols) from Refs. [5,21]. In (c) the data point indicated by the arrow was determined from our data as a cross check of the luminosity calculation.
$Q=60 \mathrm{MeV}$ by about a factor of 50 . For the $p p \rightarrow p K^{+} \Sigma^{0}$ channel, this model calculation describes the existing data set and in the case of the $p p \rightarrow p K^{+} \Lambda$ channel the underestimation of the cross section in the close-to-threshold region is about a factor of 16 being 30 times smaller than for the $\Sigma^{+}$production. At high excess energies, the $\Sigma^{+}$data points are by a factor of 3-4 below the model calculations. Previous COSY-11 hyperon production studies conclude, that final state interactions (FSI) plays an important role in the close-to-threshold $\Lambda$ production [1-3,23]. In the resonance model the FSI is not included [6,9] and therefore the deviation of the model calculations from the data points in close-to-threshold region is expected if a strong FSI is present. This effect is clearly seen for the $p p \rightarrow p K^{+} \Lambda$ and barely observed for the $p p \rightarrow p K^{+} \Sigma^{0}$ reaction channel.

In the investigation of the hyperon production in COSY-11 it was observed [1-3] that a pure 3-body phase space (PS) dependent cross section expressed as [25]:

$\sigma=K \cdot Q^{2}$

where $K$ is a normalization factor and $Q$ the excess energy cannot describe the $p p \rightarrow p K^{+} \Lambda$ data, and therefore a modification is needed which takes into account the proton-hyperon FSI. In order to describe the close-to-threshold region, the parametrisation of the excitation function including the FSI proposed by Fäldt-Wilkin [4] was used. It is expressed by:

$\sigma=C \cdot \frac{Q^{2}}{(1+\sqrt{1+Q / \varepsilon})^{2}}$,

where $C$ and $\varepsilon$ are parameters related to the FSI strength.

In Fig. 8 the cross sections for different production channels for the hyperon $\Lambda, \Sigma^{0}$ and $\Sigma^{+}$are compared to predictions of the 3-body phase space (PS, dotted line) and the 3-body phase space calculations modified by the $p Y$ FSI $(\mathrm{PS}+\mathrm{FSI}$, solid line), following equation (2) with $\epsilon$ and $C$ as free parameters.

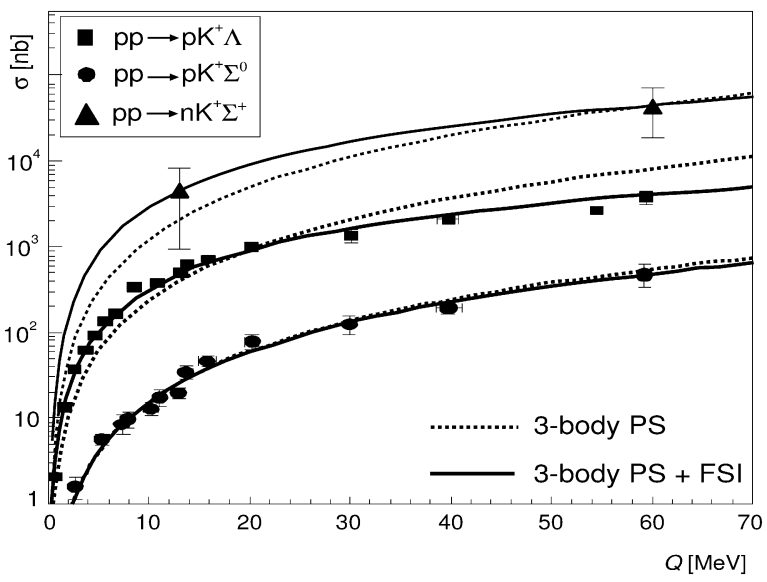

Fig. 8. The $p p \rightarrow n K^{+} \Sigma^{+}, p p \rightarrow p K^{+} \Lambda$ and $p p \rightarrow p K^{+} \Sigma^{0}$ cross sections as a function of the excess energy $Q$. Experimental data are from Refs. [1-3, 24] and from this work. The errors for the $p p \rightarrow n K^{+} \Sigma^{+}$reactions represent a sum of statistical and systematical uncertainties given in Table 2. The lines show the calculations corresponding to 3-body phase space with (solid line) and without (dashed line) final state interaction. 
These parameters are related to the scattering length $a$ and the effective range $r$ of the $p Y$ potential [4].

For the $p p \rightarrow n K^{+} \Sigma^{+}$data the resulting $\varepsilon$ and $C$ parameters are of similar values as for the $p p \rightarrow p K^{+} \Lambda$ channel. It seems that in the case of the $\Sigma^{+}$production via the $p p \rightarrow n K^{+} \Sigma^{+}$reaction a rather strong $n \Sigma^{+}$FSI is present, however, within the error bars also the curve obtained without FSI describes the two data points. Therefore for an unambiguous conclusion about the $p-\Sigma^{+}$FSI more data are needed to disentangle the reaction mechanisms and especially the role of nuclear resonances.

\section{Conclusions and perspectives}

The total cross section of the $p p \rightarrow n K^{+} \Sigma^{+}$reaction was determined at the COSY-11 detection system for excess energies of $Q=13$ and $60 \mathrm{MeV}$. However, the values established are by more than an order of magnitude larger than the expectations of any currently available model predictions.

It should be noticed that the unexpected large total $\Sigma^{+}$production cross section is somehow in line with an observation by Tan [26] who concluded that when assuming charge symmetry in $\Sigma^{+} n$ and $\Sigma^{0} p$ scattering, the contribution from the $\Sigma^{0}$ diagram is less than one seventh of the one from the $\Sigma^{+}$channel. Further, recently [27] for the case of the $\phi$ production it was suggested that a strong enhancement of the reaction amplitude towards threshold might be due to the presence of a crypto exotic baryon with hidden strangeness. Though this observation is not one-to-one conferrable to other isospin channels, in the $\Sigma^{0} p$ system no corresponding structure was observed, it might give a hint for some exotic mechanisms. Certainly the present results do not prove such a reaction process but might indicate the appearance of an interesting phenomena. In any case, present theoretical predictions of the cross sections strongly underestimate the experimental data. The adjustment of the excitation function expected from a phase space distribution including $N-Y$ FSI to the data results in parameters comparable to the $p-\Lambda$ system which may indicate a strong $n-\Sigma^{+}$interaction but due to the large systematic uncertainties the data are also consistent with a pure phase space distribution without $p-\Sigma^{+}$FSI.

Further studies of the $\Sigma^{+}$production are necessary to clarify the picture. On the experimental side additional data points should be added for which an improved event selectivity is favorable to reduce the large uncertainties introduced by the background subtraction. A $4 \pi$ detection system for neutral and charged particles which will be soon available with WASA at COSY could be used [28]. On the theoretical side an improved model has to be developed which consistently reproduces the hyperon cross section data close-to-threshold.

\section{Note added in proof}

During the evaluation of the article we have been made aware of the predictions of the excitation function of the total cross section for the $p p \rightarrow n K^{+} \Sigma^{+}$reaction which is closer to the data in comparison with the models discussed, yet still underpredict the determined total cross sections by more than an order of magnitude [29].

\section{Acknowledgements}

This work has been supported by the European Community-Access to Research Infrastructure action of the Improving Human Potential Programme, by the FFE grants (Nos. 41266606 and 41266654) from the Research Center Jülich, by the DAAD Exchange Programme (PPP-Polen), and by the Polish State Committee for Scientific Research (grant No. PB1060/P03/2004/26).

\section{References}

[1] J.T. Balewski, et al., Phys. Lett. B 420 (1998) 211.

[2] S. Sewerin, et al., Phys. Rev. Lett. 83 (1999) 682.

[3] P. Kowina, et al., Eur. Phys. J. A 22 (2004) 293.

[4] G. Fäldt, C. Wilkin, Z. Phys. A 357 (1997) 241.

[5] A. Baldini, et al., Total Cross-Section for Reactions of High-Energy Particles, Springer, Berlin, 1988.

[6] A. Sibirtsev, et al., Nucl. Phys. A 646 (1999) 427.

[7] A. Sibirtsev, et al., nucl-th/0004022.

[8] R. Shyam, et al., Phys. Rev. C 63 (2001) 022202.

[9] N. Tsushima, et al., Phys. Rev. C 59 (1999) 369.

[10] R. Shyam, hep-ph/0406297.

[11] A. Gasparian, et al., Phys. Lett. B 480 (2000) 273.

[12] A. Gasparian, et al., Nucl. Phys. A 684 (2001) 397.

[13] B. Holzenkamp, et al., Nucl. Phys. A 500 (1989) 485.

[14] R. Maier, Nucl. Instrum. Methods A 390 (1997) 1.

[15] H. Dombrowski, et al., Nucl. Instrum. Methods A 386 (1997) 228.

[16] S. Brauksiepe, et al., Nucl. Instrum. Methods A 376 (1996) 397.

[17] P. Moskal, et al., J. Phys. G 32 (2006) 629.

[18] D. Hesselbath, PhD thesis, Universität Bonn, 2000.

[19] M. Fritsch, PhD thesis, Universität Erlangen, 2002.

[20] http://www.fz-juelich.de/ikp/COSY-TOF .

[21] V. Flamino et al., Compilation of Cross Sections, CERN-HERA 84-01, 1984.

[22] A. Sibirtsev, IKP, FZ-Jülich, Germany, private communication, 2005.

[23] J. Balewski, et al., Eur. Phys. J. A 2 (1998) 99.

[24] R. Bilger, et al., Phys. Lett. B 420 (1998) 217.

[25] E. Byckling, K. Kajantie, Particle Kinematics, Wiley, London, 1973.

[26] T.H. Tan, Phys. Rev. Lett. 23 (1969) 395.

[27] A. Sibirtsev, et al., Eur. Phys. J. A 27 (2006) 263.

[28] H.-H. Adam, et al., nucl-ex/0411038.

[29] R. Shyam, Phys. Rev. C 73 (2006) 035211. 